\title{
Modenanalyse des Stegrundhohlleiters
}

\author{
Modal Analysis of a Ridged Circular Waveguide \\ Von Jürgen Marquardt und Ralf Wendel \\ Mitteilung aus dem Institut für Hochfrequenztechnik der \\ UNIVERSITATT HANNOVER
}

Übersicht:

Ein Berechnungsverfahren zur Modenanalyse des Stegrundhohlleiters mit zwei und vier rechteckigen Stegen wird vorgestellt. Der Hohlleiter wird in einen Innenbereich und Außenbereiche untergliedert. Die Außenbereiche bestehen jeweils aus einer Reihe von übereinander liegenden Halbschalen, deren Randstruktur die rechteckige Stegform approximiert. Mit Hilfe der Methode nach Kühn [1] läßt sich für jeden Teilbereich ein Potentialansatz angeben. Die Ansätze der Teilbereiche werden über die Orthogonalreihenentwicklung verknüpft und ein Gleichungssystem zur Berechnung der Eigenwerte abgeleitet. Das Eigenwertverhalten wird ausführlich dargelegt und im Zusammenhang mit Feldbildern diskutiert.

\section{Abstract:}

A modal analysis of a ridged circular waveguide is presented. The method of Kühn [1] is used to find suitable expressions for the vectorpotentials, wherefore the ridged waveguide is intersected into a lot of regions. With the mode-matching method an infinite set of linear equations is derived. This set of equations is truncated and the behaviour of the eigenvalues is shown.

Für die Dokumentation:

Orthogonalreihenentwicklung / Resonatormethode / Modenanalyse / Steghohlleiter

\section{Einleitung}

Das Einbringen von metallischen Stegen in einen Rundhohlleiter setzt die Grenzfrequenz der Grundwelle, deren elektrisches Feld parallel zu den Stegen orientiert ist, herab. Der Stegrundhohlleiter besitzt damit geringere Abmessungen als ein ungestörter Rundhohlleiter gleicher Grenzfrequenz. Eine korrekte Dimensionierung läßt sich nur mit Kenntnis des Verhaltens der Eigenwellen erreichen. Aber in der Literatur finden sich wenig Angaben. Canatan [2] berechnete mit Hilfe der Orthogonalreihenentwicklung einige Diagramme für einen Stegrundhohlleiter mit zwei und vier trapezförmigen Stegen. Chait und Sakiotis [3] sowie Chen [4] geben einige meßtechnisch, Guglielmi, Molina und Melcon [5] einige über das Finite-Elemente-Verfahren bestimmte Eigenwerte für einen Hohlleiter mit vier rechteckigen Stegen an. Die vorliegende Arbeit diskutiert erstmals ausführlich das Verhalten mit zwei und vier rechteckigen Stegen.

\section{Potentialansätze für die Raumbereiche}

Zur Modenanalyse des Stegrundhohlleiters wird der Hohlleiter in die Teilbereiche (1), (2a1), (2a2), ..., (2aN) und $(2 b 1),(2 b 2), \ldots,(2 b N)$ gemäß Bild 1 untergliedert. Es wird nur die Berechnung des Hohlleiters mit zwei Stegen wiedergegeben, der Rechnungsgang bei vier Stegen erfolgt völlig analog. Der Bereich (1) wird als Innenbereich bezeichnet. Er ist durch den Kreis mit dem Radius $\boldsymbol{R}_{\mathbf{1}}$ begrenzt, der so festgelegt ist, daß die Schnittpunkte mit der gradlinigen Stirnkante des rechteckigen Stegs um die halbe Stegbreite $B$ auseinanderliegen. Die Bereiche zwischen dem Kreis mit dem Radius $R_{1}$ und dem Außenradius des Hohlleiters $R$ werden Außenbereiche genannt und sind mit (2an) und ( $2 b n)$ indiziert, wobei $n$ ein ganzzahliger Zählindex ist,der von 0 bis

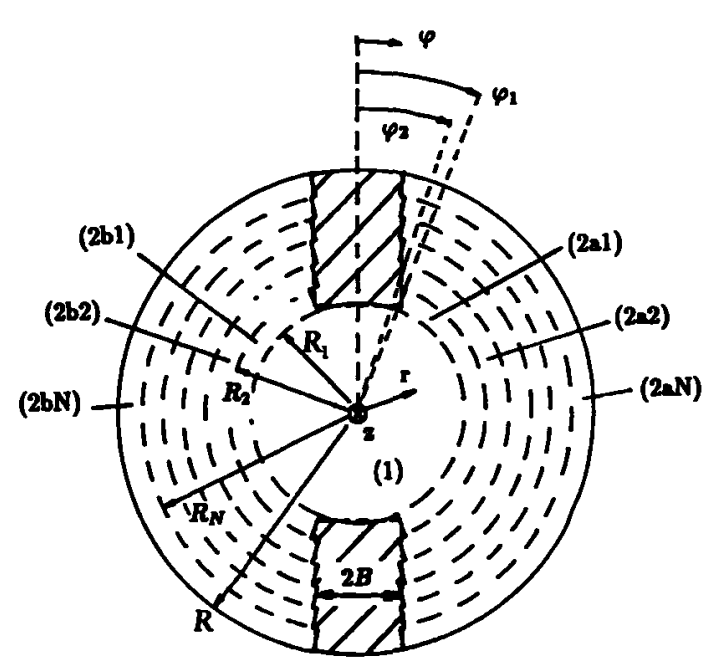

Bild 1: Stegrundhohlleiter mit zwei Stegen und dessen Untergliederung in Teilbereiche

$N$ läuft. Der $n$-te Außenbereich wird durch die Linien konstanten Winkels $\varphi_{n}$ und die Linien der konstanten Radien $R_{n}$ sowie $R_{n+1}$ begrenzt. Damit ist eine einfache Erfüllung der Randbedingungen möglich. Es gilt $\boldsymbol{R}_{N+1}=\boldsymbol{R}$. Der rechteckige Steg wird durch die Wahl von

$$
\sin \left(\varphi_{1 n}\right)=\frac{B}{R_{1}+\frac{(n-1 / 2)\left(R-R_{1}\right)}{N}}
$$

approximiert. Das Verhältnis $\frac{R_{R}}{R}$ wird als Stegtiefe bezeichnet. Die Potentialansätze in den Teilbereichen ergeben sich aus der allgemeinen Lösung der Wellengleichung in Zylin- 
derkoordinaten (z. B. [6]). Das Potential setzt sich aus unendlich vielen Teilpotentialen mit verschiedenen Eigenwerten zusammen. Die Felder der E-Wellen werden aus dem magnetischen Vektorpotential $\vec{A}=\left(0,0, A_{z}\right)$, die der H-Wellen aus dem elektrischen Vektorpotential $\vec{F}=\left(0,0, F_{z}\right)$ abgeleitet. Der Potentialansatz im Innenbereich lautet für die $i$-te Eigenwelle

$$
\begin{aligned}
& A_{i}^{(1)}=\sum_{m=0}^{\infty} C_{m e i}^{(1)} \mathrm{J}_{m}\left(k_{\text {rei }} r\right)\left\{\begin{array}{l}
\operatorname{sim}(m \varphi) \\
\cos (m \varphi)
\end{array}\right\} \\
& F_{i}^{(1)}=\sum_{m=0}^{\infty} C_{m h i}^{(1)} \mathrm{J}_{m}\left(k_{\text {mi }} r\right)\left\{\begin{array}{l}
\operatorname{sim}(m \varphi) \\
\cos (m \varphi)
\end{array}\right\}
\end{aligned}
$$

mit

$$
\operatorname{sim}(m \varphi)= \begin{cases}1 & m=0 \\ \sin (m \varphi) & \text { sonst }\end{cases}
$$

und

$$
m=0,1,2,3, \ldots
$$

Der Index e kennzeichnet die E-Wellen, der Index $h$ die $H$-Wellen. Die Grenzfrequenz der $\boldsymbol{i}$-ten Eigenwelle ist über

$$
f_{\mathrm{e}}=\frac{1}{2 \pi R} \frac{k_{r i}}{\sqrt{\mu \varepsilon}}
$$

mit dem Eigenwert $k_{r i}$ verknüpft. Ein Ansatz für das Potential im $n$-ten Außenbereich wird mit Hilfe der Resonatormethode [1] erstellt. Nach dieser ist ein Ansatz möglich, der sich aus zwei Teilansätzen zusammensetzt, wobei beim ersten Teilansatz die Grenze zum $(n+1)$-ten Bereich und beim zweiten Teilansatz die Grenze zum ( $\boldsymbol{n}$-1)-ten Bereich als ideal elektrisch leitend gedacht wird. Damit ergibt sich

$$
\begin{aligned}
A_{i}^{(2 p n)}= & \sum_{u=1}^{\infty}\left[C_{u e i}^{(2 p n)} X_{k_{\text {pru }}}\left(k_{r e i} r\right)\right. \\
& \left.+D_{u e i}^{(2 p n)} Z_{k_{\text {pru }}}\left(k_{r e i} r\right)\right] \sin \left(k_{\varphi n u}\left(\varphi-\varphi_{p n}\right)\right) \\
F_{i}^{(2 p n)}= & \sum_{u=1}^{\infty}\left[C_{u h i}^{(2 p n)} X_{k_{\text {quu }}}\left(k_{r h i} r\right)\right. \\
& \left.+D_{u h i}^{(2 p n)} Z_{k_{\text {quu }}}\left(k_{r \mathrm{ri}} r\right)\right] \cos \left(k_{\varphi n u}\left(\varphi-\varphi_{p n}\right)\right)
\end{aligned}
$$

mit

$$
\begin{aligned}
& X_{k_{\text {eru }}}\left(k_{\text {rei }} r\right)=J_{k_{\text {quu }}}\left(k_{\text {rei }} r\right) \\
& -\frac{J_{k_{\text {enu }}}\left(k_{\text {rei }} R_{n+1}\right)}{Y_{k_{\text {emu }}}\left(k_{\text {rei }} R_{n+1}\right)} Y_{k_{\text {smu }}}\left(k_{\text {rei }} r\right) \\
& Z_{k_{\text {pax }}}\left(k_{\text {rei }} r\right)=J_{k_{\text {pre }}}\left(k_{\text {rei }} r\right) \\
& -\frac{J_{k_{\text {priu }}}\left(k_{\text {rei }} R_{n}\right)}{Y_{k_{\text {put }}}\left(k_{\text {rei }} R_{n}\right)} Y_{k_{\text {owi }}}\left(k_{\text {rei }} r\right)
\end{aligned}
$$

$$
\begin{aligned}
X_{k_{\text {pru }}}\left(k_{\mathrm{mi} i} r\right)= & \mathrm{J}_{k_{\text {pru }}}\left(k_{\mathrm{mi} i} r\right) \\
& -\frac{J_{k_{\text {qui }}}\left(k_{\mathrm{mi} i} R_{n+1}\right)}{Y_{k_{\text {qui }}}\left(k_{\mathrm{mi} i} R_{n+1}\right)} Y_{k_{\text {pru }}}\left(k_{\mathrm{ri} i} r\right)
\end{aligned}
$$

$$
\begin{aligned}
& X_{k_{\text {prua }}}\left(k_{r \mathrm{hi}} r\right)=\mathrm{J}_{k_{\text {qriu }}}\left(k_{\mathrm{rhi}} r\right)
\end{aligned}
$$

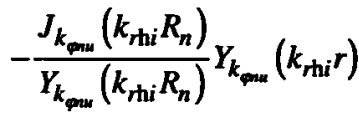

und

$$
\varphi_{p n}=\left\{\begin{array}{ll}
\varphi_{1 n} & p=a \\
\varphi_{1 n}+\pi & p=b
\end{array} .\right.
$$

Die Abkürzung (2pn) steht für den Teilbereich ( $2 a n$ ) bzw. (2bn). Die Randbedingungen auf den Stegflanken werden durch

$$
k_{\varphi n u}=\frac{u \pi}{\pi-2 \varphi_{1 n}}
$$

erfüllt. Die Indizes $\boldsymbol{k}_{\text {甲nu }}$ der Besselfunktionen sind nicht ganzzahlig und in jedem Teilbereich des Außenbereiches verschieden. Der Index $u$ ist ganzzahlig, er beginnt bei H-Wellen bei 0 und bei E-Wellen bei 1 . Der Ansatz $X_{k_{\text {mun }}}$ mit der Linearkombination aus Besselfunktionen 1. und 2. Ordnung, der den Koeffizienten $C_{u e i}^{(2 p n)}$ und $C_{u h i}^{(2 p n)}$ zugeordnet ist, bewirkt ein Verschwinden des tangentialen elektrischen und normalen magnetischen Feldes auf der Radiuslinie $\boldsymbol{R}_{n+1}$. Entsprechend verschwinden beim Ansatz $Z_{k_{\text {can }}}$ - dieser ist den Koeffizienten $D_{u e t}^{(2 p n)}$ und $D_{u h i}^{(2 p n)}$ zugeordnet - die genannten Felder auf Radiuslinie $R_{n}$. Im äußersten Teilbereich ( $\left.2 p N\right)$ gilt $D_{u \in i}^{(2 p n)}=D_{u \mathrm{hi}}^{(2 p n)}=0$.

\section{Anpassung der Ansätze an den Grenz- flächen}

Die Ansätze zweier aneinander grenzender Teilbereiche werden über die Forderung nach Stetigkeit des Potentials und Stetigkeit der Ableitung des Potentials bezüglich des Radius verknüpft. Auf diesen Beziehungen basierend liefert die Entwicklung der Koeffizienten im Innenbereich (1) aus denen im Teilbereich (2a1) und (2b1)

$$
\left[C^{(1)}\right]=\left[M_{1}\right]\left[C^{(2 a 1)}\right]+\left[M_{2}\right]\left[C^{(2 b 1)}\right]
$$

Die Entwicklung der Koeffizienten in den Außenbereichen (2a1) bzw. (2b1) aus denen im Innenbereich ergibt:

$$
\begin{aligned}
& {\left[M_{3}\right]\left[C^{(2 a 1)}\right]+\left[M_{4}\right]\left[D^{(2 a 1)}\right]=\left[M_{5}\right]\left[C^{(1)}\right]} \\
& {\left[M_{6}\right]\left[C^{(2 b 1)}\right]+\left[M_{7}\right]\left[D^{(2 b 1)}\right]=\left[M_{8}\right]\left[C^{(1)}\right] .}
\end{aligned}
$$

Die Ausdrücke $\left[C^{(1)}\right],\left[C^{(2 a 1)}\right]$ usw. sind die Spaltenvektoren der Koeffizienten $C^{(1)}, C^{(2 a 1)}$ usw. Die Matrizen $\left[M_{1}\right],\left[M_{2}\right]$, $\left[M_{5}\right]$ und $\left[M_{8}\right]$ enthalten Koppelintegrale, deren Integranden aus dem Produkt zweier trigonometrischer Funktionen bestehen. Die übrigen Matrizen $\left[M_{i}\right]$ sind Diagonalmatrizen mit konstanten Faktoren. Die Gleichungen (15), (16) und (17) lassen sich zu dem Gleichungssystem 


$$
\left[M_{9}\right]\left[\begin{array}{c}
C^{(1)} \\
C^{(2 a 1)} \\
C^{(2 b 1)} \\
D^{(2 a 1)} \\
D^{(2 b 1)}
\end{array}\right]=[0]
$$

zusammenfassen. Aus der wechselseitigen Entwicklung der Koeffizienten an der Grenzfläche zwischen dem $\boldsymbol{n}$-ten und $(n+1)$-ten Teilbereich folgt

$$
\begin{aligned}
& {\left[M_{10}\right]\left[\begin{array}{l}
C^{(2 p n)} \\
D^{(2 p n)}
\end{array}\right]=\left[M_{11}\right]\left[\begin{array}{l}
C^{(2 p n+1)} \\
D^{(2 p n+1)}
\end{array}\right]} \\
& {\left[M_{12}\right]\left[D^{(2 p n+1)}\right]=\left[M_{13}\right]\left[C^{(2 p n)}\right]}
\end{aligned}
$$

Der Index 2pn steht dabei stellvertretend für die Bereiche $2 a n$ bzw. $2 b n$. Durch (19) und (20) lassen sich die Koeffizienten des n-ten Teilbereiches durch die des $(n+1)$-ten Teilbereiches ausdrücken:

$$
\left[\begin{array}{l}
C^{(2 p n+1)} \\
D^{(2 p n+1)}
\end{array}\right]=\left[M_{14}\right]\left[\begin{array}{l}
C^{(2 p n)} \\
D^{(2 p n)}
\end{array}\right]
$$

Die Umformung erfordert eine Matrizeninversion, die nur möglich ist, wenn in jedem Teilbereich der Außenbereiche gleich viele Funktionen angesetzt werden. Durch fortgesetztes Einsetzen $\cdot$.12 (21) in (18) ergibt sich\}

$$
\operatorname{la}{ }_{15}\left[\begin{array}{c}
C^{(1)} \\
C^{(2 a N)} \\
C^{(2 b N)}
\end{array}\right]=[0]
$$

Die Koeffizienten $D^{(2 p N)}$ treten nicht auf, da sie im $N$-ten Teilbereich nicht vorhanden sind. Das Gleichungssystem (22) enthält so viele Gleichungen wie Unbekannte. Der zur $i$-ten Eigenwelle gehörige Eigenwert ist in nichtlinearer Form in der Matrix $\left[M_{15}\right]$ enthalten. Eine nichttriviale Lösung des Systems erhält man, wenn die Forderung

$$
\operatorname{det}\left[M_{15}\right]=0
$$

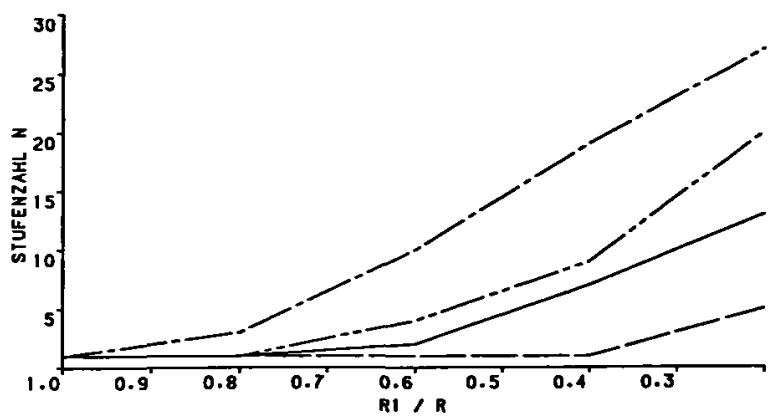

Bild 2: Stufenzahl N in Abhängigkeit von der Stegtiefe für gegebene Genauigkeit

$$
B / R=0,01 \quad 0,1 \%
$$$$
\begin{array}{lll}
- & B / R=0,01 & 0,5 \% \\
--D- & B / R=0,14 & 0,1 \%
\end{array}
$$$$
\text { - - - - B B R }=0,14 \quad 0,5 \%
$$

erfüllt wird. Zur Bestimmung des gesuchten Eigenwertes läßt sich die Determinante von $\left[M_{15}\right]$ schrittweise auf Vorzeichenwechsel abtasten und eine gefundene Nullstelle bis auf eine bestimmte Nachkommazahl eingrenzen. Die Genauigkeit der Lösung hängt von der absoluten Zahl der angesetzten Funktionen sowie von der Zahl der Teilbereiche ab. Weil das Verfahren extrem schnell konvergiert, liefert der Ansatz von nur 10 Funktionen in der Regel bereits eine Genauigkeit von $1 \%$. Die Zahl der für eine vorgegebene Genauigkeit notwendigen Teilbereiche kann Bild 2 entnommen werden. Zur Ermittlung des Bildes wurden 25 Funktionen angesetzt. Bis zur Stegtiefe von $\frac{R_{1}}{R}=0,4$ lassen sich schmale rechteckige Stege $\left(\frac{B}{R}=0,01\right)^{R}$ mit nur einem Außenbereich mit einer Genauigkett von $0,5 \%$ berechnen, $d$. h. der rechteckige Steg darf durch einen trapezförmigen Steg angenähert werden.

\section{Modenverhalten}

Die Moden im Stegrundhohlleiter werden in Anlehnung an den Rundhohlleiter ohne Steg mit $\mathrm{H}_{11}, \mathrm{H}_{21}, \ldots$ und $\mathrm{E}_{01}$, $E_{11}, \ldots$ bezeichnet. Je nach Ansatz wird bei zwei Stegen in die Modenbezeichnung ein $\mathrm{C}$ für den cos-Ansatz und ein $\mathrm{S}$ für den sim-Ansatz eingefügt. Ein HS- und ein EC-Mode weisen ein elektrisches Feld auf, das parallel zu den Stegen orientiert ist; bei HC- und ES-Moden ist die Orientierung senkrecht. Man beachte, daß die so eingeführte Bezeichnung der
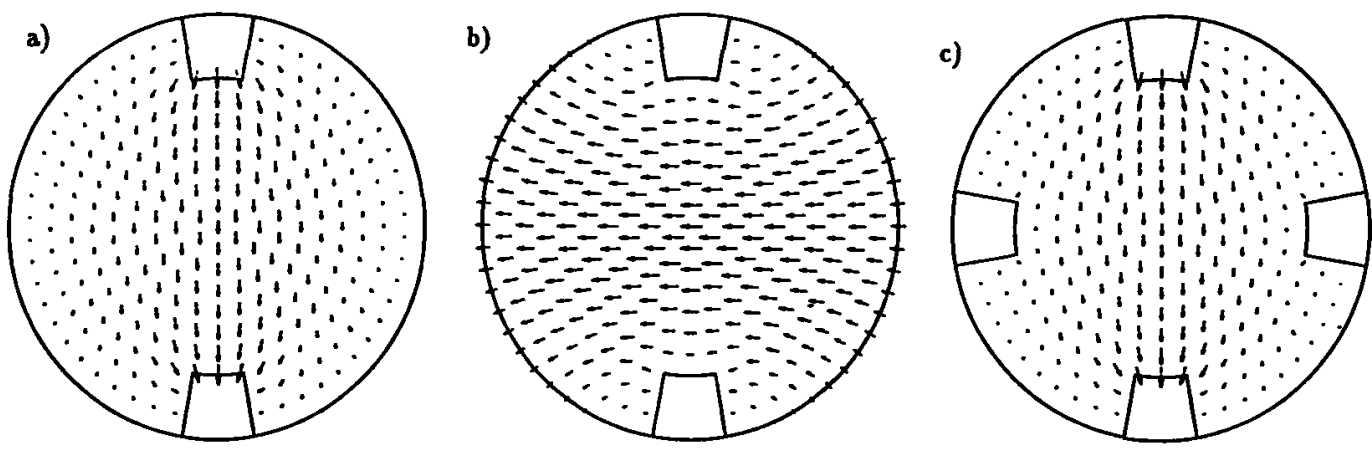

a) $\mathbf{H} \mathbf{S}_{11}$-Mode, 2 Stege

Bild 3: Verlauf der elektrischen Feldlinien im Hohlleiterquerschnitt

b) $\mathrm{HC}_{11}$-Mode, 2 Stege

c) $\mathrm{H}_{11}$-Mode, 4 Stege 
Stegrundhohlleitermoden aus dem Grenzfall des ungestörten Rundhohlleiters definiert ist, und nur im Grenzfall direkten Bezug zur Radius- und Winkelabhängigkeit hat. Bei einer endlichen Stegtiefe ist ein Stegrundhohlleitermode nicht wie im ungestörten Rundhohlleiter durch nur eine Umfangs- und Radiusabhängigkeit, sondern durch die Überlagerung theoretisch unendlich vieler Umfangs- und Radiusabhängigkeiten gekennzeichnet. Bild 3 zeigt die Feldbilder für die Grundmoden für den Sonderfall trapezförmiger Stege. Für den $\mathrm{HC}_{11}$-Mode liegen die Stege äquipotential zum elektrischen Feld und die Feldverteilung wird durch die Stege

a)

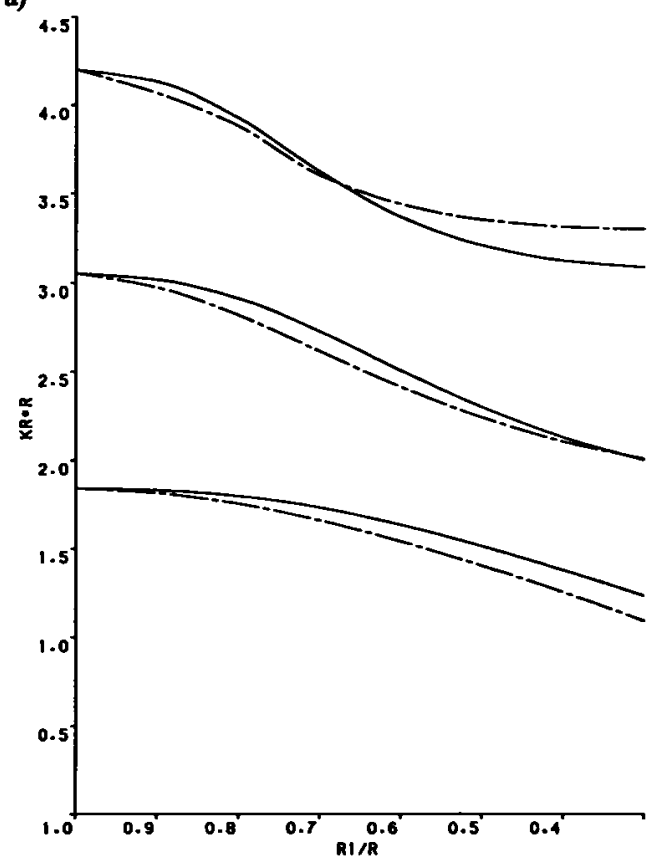

c)

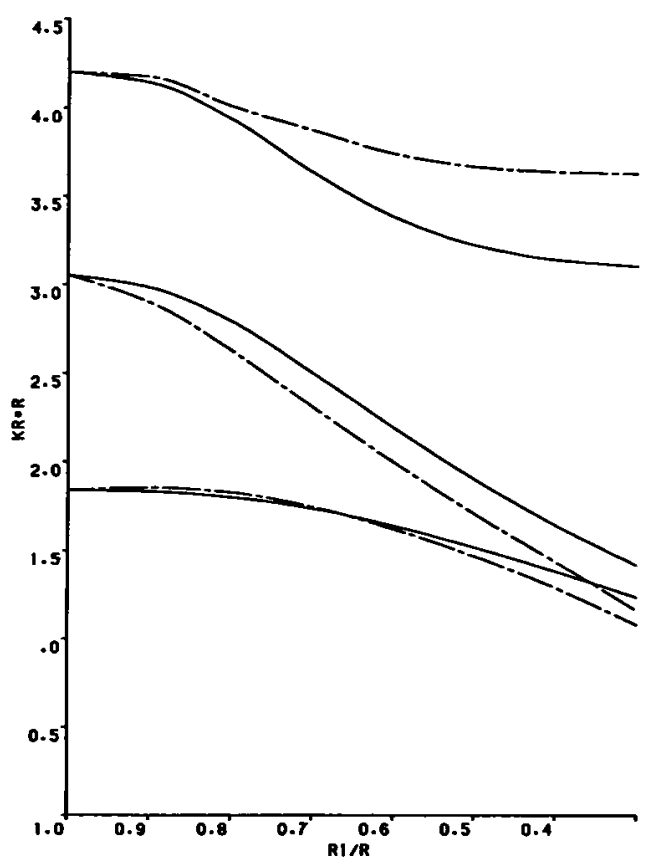

nur wenig beeinflußt. Senkrecht zum Feld des $\mathrm{HC}_{11}$-Modes ist das Feld des $\mathrm{HS}_{11}$-Modes orientiert. Es wird mit wachsender Stegtiefe kurzgeschlossen. Bei vier Stegen zeigt sich ein Feldbild, das der Überlagerung von $\mathrm{HS}_{11}$-und $\mathbf{H C}_{11^{-}}$ Moden entspricht. Diese Feldbilder veranschaulichen auch die Abhängigkeit der Eigenwerte (und damit der Grenzfrequenzen) von den Stegabmessungen.

In Bild 4 sind die Eigenwerte $k_{r} R$ in Abhängigkeit von der normierten Stegtiefe dargestellt. Die Eigenwerte der HSModen verkleinern sich mit steigender Tiefe der Stege

b)

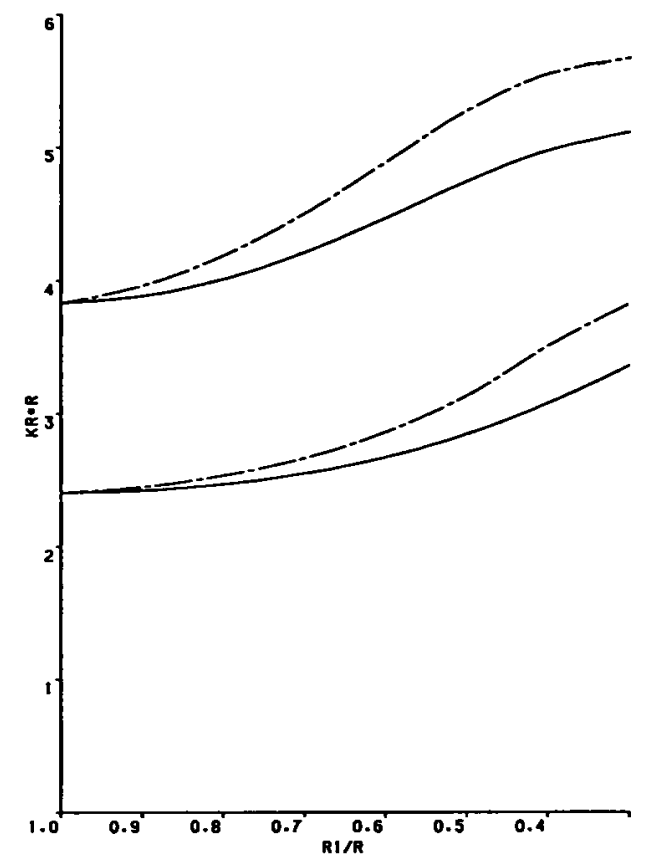

d)

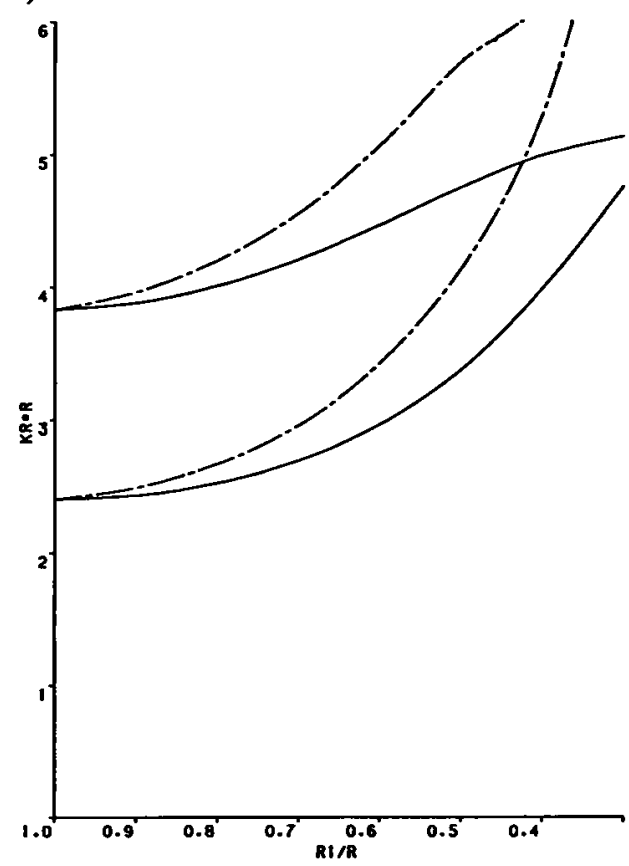

Bild 4: Eigenwertverhalten der Stegrundhohlleitermoden

a) HS-Moden, 2 Stege

$\longrightarrow B / R=0,01,--\longrightarrow B / R=0,14$

b) EC-Moden, 2 Stege

c) H-Moden, 4 Stege

d) E-Moden, 4 Stege 
(Bild 4a). Der HS HI -Grundmode weist deshalb eine kleinere Grenzfrequenz und einen kleineren Wellenwiderstand auf als der Grundmode im ungestörten Rundhohlleiter mit gleichem Radius. Die Eigenwerte der EC-Moden steigen dagegen mit zunehmender Tiefe an, weil die Feldverteilung des glatten Rundhohlleiters durch die Stege stark gestört wird (Bild 4b). Das Abfallen des Eigenwerts des $\mathrm{HS}_{11}$-Modes und der Anstieg des Eigenwerts des EC $_{01}$-Modes führt dazu, daß sich mit steigender Stegtiefe der Bereich, in dem nur ein Stegrundhohlleitermode ausbreitungsfähig ist, vergrößert. Bei weit in den Hohlleiter hineinragenden Stegen begrenzt allerdings nicht mehr der $\mathrm{EC}_{01}$-Mode sondern der $\mathrm{HS}_{21}$-Mode den Eindeutigkeitsbereich. Mit zwei Stegen der normierten Stegtiefe 0,6 bis 0,7 läßt sich der eindeutige Betriebsfrequenzbereich des glatten Rundhohlleiters verdoppeln. Bei vier Stegen nimmt die Größe des Eindeutigkeitsbereiches durch die Annäherung des $\mathrm{HS}_{21}$-Modes an den $\mathrm{HS}_{11}$-Mode wieder ab (Bilder $4 c$ und 4d), so daß keine Erweiterung der eindeutigen Nutzung erreicht wird. Die Vergleiche der hier berechneten Eigenwerte mit Literaturwerten aus [5] sowie mit Meßwerten an einem Resonator [7] zeigen eine maximale Abweichnung von $1 \%$.

\section{Zusammenfassung}

Das Modenverhalten des Stegrundhohlleiters wurde unter Zuhilfenahme der Methode von Kühn mit dem Verfahren der
Orthogonalreihenentwicklung berechnet. Die Modenverläufe wurden dargestellt und im Zusammenhang mit Feldbildern erläutert. Das vorgestellte Verfahren konvergiert extrem schnell und zeigt hohe Genauigkeit.

\section{Literatur:}

[1] Kähn, E.: A-Mode-Matching Method for Solving Field Problems in Waveguides and Resonator Circuits. Archiv. d. elektr. Übertr., 27 (1973) 12, S. 511-518.

[2] Canatan, F.: Cutoff Wavenumbers of Ridged Circular Waveguides via RitzGalerkin Approach. Electronics Letters, Vol. 25 (1989) No. 16.

[3] Chait, H.; Sakiotis, N. G.: Broad-End Ferrite Rotators Using QuadruplyRidged Circular Waveguide. IRE Trans. Microwave Theory and Tech., Jan. 1959, S. 38-41.

[4] Chen, C.-C.: Quadruply Ridge-Loaded Circular Waveguide Phased Arrays. IEEE Trans. Antennas and Propagation, Vol. MTT-22 (1974) No. 5, S. 481-483.

[5] Guglielmi, M.; Molina, R. C.; Melcon, A.: Dual-Mode Circular Waveguide Filters Without Tuning Screws. IEEE Microwave and Guided Wave Letters, Vol. 2 (1992) No. 11, S. 457-458.

[6] Unger, H. G.: Elektromagnetische Theorie fïr die Hochfrequenztechnik Teil 1 und 2. Hüthig Veriag, Heidelberg, 2. Aufl. 1989.

[7] Wendel, R.: Berechnung von Hohlleiterspríngen und Stegen im Rundhohlleiter. Dissertation am Institut für Hochfrequenztechnik, Universität Hannover 1994.
Prof. Dr.-Ing. J. Marquardt

Dr.-Ing. R. Wendel

Appelstr. 9a

(Eingegangen am 4.4.1995)

\section{Neues aus Forschung, Industrie und Wirtschaft}

\section{Neuer Controller gleicht Schwankungen der Versorgungs- spannung aus}

Texas Instruments (TI) hat seine Power+ Intelligence Familie um einen Baustein erweitert: Der TPIC2101 ist ein Controller für bürstenbehaftete Gleichstrommotoren, der Schwankungen der Versorgungsspannung ausgleicht und für eine konstante Spannungsbelastung sorgt.

Bei der Regelung von Elektromotoren kommt es vor allem darauf an, Schwankungen der Versorgungsspannung weitestgehend auszuschalten. Der neue Pre-FET-Treiberbaustein TPIC2101 bietet einen einfachen und kostengünstigen Weg, eine konstante Lastspannung in Gleichstrommotor-Regelungen zu gewährleisten. $\mathrm{Er}$ ist ein 50 mA Einphasen-Low-Side-Pre-FET-Treiber mit Pulsbreitenmodulation (PWM) für eine Betriebstemperatur zwischen $-40^{\circ} \mathrm{C}$ und $105^{\circ} \mathrm{C}$. Er ermöglicht eine konstante Drehzahlregelung für unidirektionale bürstenbehaftete Gleichstrommotoren vor allem in Anwendungen, bei denen die Spannungsversorgung im Normalbetrieb schwankt. Der TPIC2101 gleicht die Drehzahlschwankungen dadurch aus, daß der PWM-Lastzyklus umgekehrt proportional zu den Schwankungen der Versorgungsspannung im
Bereich 8 V bis 16 V modifiziert wird. Durch Anpassung des Steuerstroms an den FET wird eine konstante Effektivspannung und damit eine konstante Drehzahl erreicht.

Für die automatische Drehzahlregelung kann der TPIC2101 direkt mit einem Mikrocontroller gekoppelt werden; eine manuelle Drehzahlregelung über die Signale eines Regelwiderstandes ist ebenfalls möglich.

Der TPIC2101 bietet drei Betriebszustände. Im Normalzustand wird der Baustein durch jeden Drehzahlbefehl aktiviert. Bei Fehlen eines Eingangssignals bzw. bei Unterspannung wird der Baustein in einen Bereitschaftszustand $(<200 \mu \mathrm{A})$ geschaltet; auf diese Weise wird die Leistungsaufnahme möglichst gering gehalten. Um den externen FET und die Last zu schützen, wird der Baustein bei Überspannung oder bei Vorliegen eines Fehlerstroms in einen Fehlermodus geschaltet.

Um die Anfahrbelastung niedrig zu halten und so die Lebensdauer des Motors zu verlängern, wird das PWM-Ausgangssignal automatisch hochgefahren, so daß ein "weicher" Motorstart gewährleistet ist. Der TPIC2101 wird in einem platzsparenden DIP- oder SO-Gehäuse mit 14 Pins geliefert. 